\title{
Effect of aspirin and non-steroidal anti-inflammatory drugs on colorectal adenomas: case-control study of subjects participating in the Nottingham faecal occult blood screening programme
}

\author{
R F A Logan, J Little, P G Hawtin, J D Hardcastle
}

\begin{abstract}
Objective-To examine the relation between the use of aspirin and non-steroidal anti-inflammatory drugs and the presence of asymptomatic colorectal adenomas.

Design-Case-control study of subjects participating in a randomised controlled trial of faecal occult blood screening for colorectal cancer. Data on analgesics and other drugs were obtained from a questionnaire which was mainly concerned with diet and was administered by an interviewer.

Setting-Nottingham.

Subjects -147 patients with positive results in faecal occult blood tests who were found to have colorectal adenomas (cases), 153 age and sex matched control subjects with negative results in such tests (negative controls), and 176 control subjects with positive results in the tests who were found not to have colorectal adenomas (positive controls).
\end{abstract}

Main outcome measures-Relative risk of developing colorectal adenomas according to frequency and duration of use of analgesics.

Results-Cases reported taking less aspirin and non-steroidal anti-inflammatory drugs than the negative controls, with the estimated relative risk for any use being $0.49(95 \%$ confidence interval 0.3 to $0 \cdot 8)$. The inverse association was less strong when cases were compared with the positive controls $(0.66(0.4$ to 1.1$))$. The association was specific for aspirin and non-steroidal anti-inflammatory drugs there being no association with paracetamol or other drugs. Prescribed use of non-steroidal antiinflammatory drugs for longer than five years was associated with the lowest risk $(0.21(0.1$ to 0.8$))$, although the numbers reporting prolonged prescribed use were small.

Conclusions-These findings support the hypothesis that aspirin and non-steroidal antiinflammatory drug use protects against the development of colorectal neoplasia.

\section{Introduction}

Three lines of evidence suggest that regular ingestion of aspirin or other non-steroidal anti-inflammatory drugs might protect against the development of colorectal neoplasia. Firstly, several non-steroidal antiinflammatory drugs, including aspirin, inhibit the growth of chemically induced tumours of the colon in rats and mice. ${ }^{1-9}$ Secondly, the non-steroidal antiinflammatory drug sulindac causes the regression of residual rectal polyps in patients with familial adenomatous polyposis and an ileorectal anastomosis. ${ }^{10-13}$ Finally, in three epidemiological studies the use of aspirin and non-steroidal anti-inflammatory drug was associated with an approximate halving of the risk of developing or dying from colonic or colorectal cancer. ${ }^{1416}$ However, a fourth, smaller study found no association between aspirin use and the development of colonic cancer. ${ }^{17}$

As most colorectal cancers are thought to develop from colorectal adenomas, we analysed data on analgesic use collected during a recent case-control study of colorectal adenomas and diet. ${ }^{18}$

Subjects and methods RECRUITMENT OF SUBJECTS

Subjects were recruited from among those who had had a faecal occult blood test in a randomised trial of screening for colorectal cancer in Nottingham. ${ }^{19}$ Cases were subjects found to have adenomatous colorectal polyps after a test gave positive results. Two types of control were recruited for each case: (a) subjects matched for age and sex who had negative test results (negative controls) and $(b)$ subjects who had positive results on screening but were found to be free of adenomas and carcinomas on examination by colonoscopy or sigmoidoscopy and barium enema (positive controls). The age and sex matched controls were obtained by asking the first subject in the screening trial records with the appropriate characteristics to participate; as the trial was carried out in various general practices, cases and controls effectively came from the same practice.

Cases and controls were invited to participate in this study once any hospital investigation and treatment as a consequence of screening had been completed. The screening trial from which the subjects were recruited started in 1981, and we included subjects who had completed faecal occult blood tests up to 30 June 1988 . We interviewed subjects between November 1985 and September 1988.

\section{DATA COLLECTION}

Information on dietary habits, height and weight, occupational history, leisure activity, demographic factors, and medical history was obtained by an interview conducted at the subject's home by specially trained interviewers. Data on analgesic consumption were based on the responses to two questions: "In the past five years have you taken any medicines or drugs prescribed by your doctor on a regular basis? If so what?" and "Do you ever take aspirin or any other pain killer? If so what?" Subjects were asked to recall their drug consumption during the five years before they had a faecal occult blood test, and drugs that had been taken only since the tests were ignored. As far as possible the name, dose, duration of use, and frequency of use of each drug was then recorded. Duration of use of prescribed drugs was recorded as the number of months of regular use, and use for a total of less than three months was ignored. Frequency of non-
Correspondence to: Dr Logan.

BMF 1993;307:285-9 
prescribed use was coded as once or twice a year only, more than once or twice a year but less than monthly, more than monthly but less than weekly, weekly but not daily, and daily.

ANALYSIS

The unmatched estimated relative risks are odds ratios calculated by the Mantel-Haenszel technique with the SEARCH package and by unconditional logistic regression with the GLIM package. ${ }^{2021}$ When appropriate $\chi^{2}$ tests for trend were performed by logistic regression analysis. A matched analysis by conditional logistic regression was also performed on the data from the 129 completed matched pairs with the PECAN program. ${ }^{22}$ The matched analysis was adjusted for social class and the unmatched analyses for age, sex, and social class. Other potential confounding factors were identified by examining separately the relation between the use of non-steroidal antiinflammatory drugs and previous illness, drug usage, and selected variables concerned with diet and physical activity. ${ }^{18}$

\section{Results}

COMPOSITION OF THE STUDY GROUPS

Between January 1981 and June 1988, 606 subjects in the trial had positive results in faecal occult blood tests. In 222 a polyp, thought to be adenomatous, was found. Of these, 29 could not be approached because they had died, moved away, or were regarded as unfit to be interviewed. Of the remaining $193,169(88 \%)$ agreed to be interviewed, but in 22 the polyp was either not retrieved (nine) or not adenomatous on histological examination (13)

Of the control subjects with negative results in faecal occult blood tests, 41 could not be approached because they had died (seven), moved away (11), or were regarded as unfit to be interviewed (23). Of the 169 eventually approached, $153(91 \%)$ agreed to be interviewed. A total of 384 subjects with positive screening results remained to act as potential controls. Of these, 62 were excluded for logistic reasons from two practices outside Nottingham which had used a faecal

TABLE I-Sociodemographic and other characteristics of cases and controls. Values are numbers (percentages) of subjects unless stated otherwise

\begin{tabular}{lccc}
\hline & \multicolumn{2}{c}{ Controls } \\
\cline { 3 - 4 } & Cases (n=147) & Negative (n-153) & Positive (n=176) \\
\cline { 3 - 4 } Men & $91(62)$ & $94(61)$ & $86(49)$ \\
Mean (SD) age (years): & $65(7)$ & $65(7)$ & $63(7)$ \\
Men & $67(8)$ & $68(7)$ & $64(8)$ \\
Women & $11(8)$ & $6(4)$ & $8(5)$ \\
Social class of longest occupation ${ }^{\star}:$ & $16(11)$ & $29(19)$ & $24(14)$ \\
I & $30(20)$ & $41(27)$ & $38(22)$ \\
II & $54(37)$ & $55(36)$ & $65(37)$ \\
IIIN & $25(17)$ & $13(9)$ & $30(17)$ \\
IIIM & $3(2)$ & $3(2)$ & $4(2)$ \\
IV & $8(5)$ & $6(4)$ & $7(4)$ \\
Other & $101(69)$ & $110(72)$ & $117(67)$ \\
Subjects who smoked at some time & & & \\
\hline
\end{tabular}

occult blood test giving a high false positive rate and 37 because they could not be approached for reasons similar to the cases. ${ }^{23} \mathrm{~A}$ further 68 were found to have cancers, of whom 46 were interviewed as part of a parallel study of diet and colorectal cancer in which data were collected until April 1992. Of the remaining $217,176(81 \%)$ agreed to be interviewed.

Table I summarises the sociodemographic characteristics of the three groups. The distributions according to the socioeconomic state associated with the job held for the longest period differed between cases and controls with negative results in faecal occult blood tests $\left(\chi^{2}=10 \cdot 9, \mathrm{df}=6, \mathrm{p}=0.09 ; \chi^{2}\right.$ for trend $=2 \cdot 3, \mathrm{df}=1$, $p>0 \cdot 1)$. When adjustment was made for this measure, no associations with other measures of socioeconomic state were found. There were no notable differences between the groups in mean age on leaving school, level of education since leaving school, marital state, length of residence in the Nottingham area, or place of birth. The control group with positive screening results was slightly younger and contained more women than the other two groups.

SIZE, HISTOLOGICAL TYPE, AND MULTIPLICITY OF ADENOMAS

The adenoma was in the rectum, rectosigmoid, or descending colon in 117 of the 122 cases in which site was recorded. It was recorded as being less than $1 \mathrm{~cm}$ in maximum diameter or small in 42 cases, as $1-1.9 \mathrm{~cm}$ or medium in 70 cases, and as $2 \mathrm{~cm}$ or more in maximum diameter or large in 30 cases. In 34 cases more than one adenoma was found and 72 had villous or tubulovillous adenomas.

\section{TYPES OF ANALGESIC USED}

Table II shows that cases reported taking less aspirin and other non-steroidal anti-inflammatory drugs than either control group. In contrast cases reported taking slightly more paracetamol than both the control groups. A matched analysis of the data for the 129 completed pairs gave very similar results.

As the relative risk estimates associated with aspirin and with other non-steroidal anti-inflammatory drugs were similar they were combined for subsequent analyses. The inverse association with non-steroidal anti-inflammatory drug use persisted irrespective of paracetamol use, although the numbers of subjects taking both non-steroidal anti-inflammatory drugs and paracetamol were small (table II). A total of $40(27 \%)$ cases reported taking non-steroidal anti-inflammatory drugs sometimes compared with $65(42 \%)$ of the controls with negative screening results and $63(36 \%)$ of those with positive results, giving unmatched estimated relative risks of $0.49(95 \%$ confidence interval 0.3 to 0.8$)$ and $0.66(0.4$ to 1.1$)$ respectively.

DURATION AND FREQUENCY OF ANALGESIC USE

When prescribed non-steroidal anti-inflammatory drugs were considered (table III) continuous use for five years or more was associated with lower risks of having an adenoma than shorter periods of continuous

TABLE II-Colorectal adenomas and type of analgesic taken among cases and controls

\begin{tabular}{|c|c|c|c|c|c|c|}
\hline & \multirow[b]{2}{*}{ Cases $(n=147)$} & \multirow{2}{*}{$\begin{array}{l}\text { Negative } \\
\text { controls } \\
(n=153)\end{array}$} & \multicolumn{2}{|c|}{ Relative risk ( $95 \%$ confidence interval) } & \multirow{2}{*}{$\begin{array}{l}\text { Positive } \\
\text { controls } \\
(n=176)\end{array}$} & \multirow{2}{*}{$\begin{array}{l}\text { Adjusted relative risk } \\
\text { (95\% confidence } \\
\text { interval)* }\end{array}$} \\
\hline & & & Adjusted ${ }^{\star}$ & Matched & & \\
\hline \multicolumn{7}{|l|}{ Any use off: } \\
\hline Aspirin & 27 & 43 & $0.57(0.3$ to 1.0$)$ & $0.55(0.3$ to 1.1$)$ & 43 & $0.72(0.4$ to 1.3$)$ \\
\hline Other NSAIDs & 15 & 27 & $0.50(0.3$ to 1.0$)$ & $0.56(0.3$ to 1.2$)$ & 23 & $0.69(0.3$ to 1.4$)$ \\
\hline Paracetamol & 79 & 66 & $1.51(0.9$ to 2.4$)$ & $1.65(0.9$ to 3.0$)$ & 95 & $1.11(0.7$ to 1.8$)$ \\
\hline \multirow{5}{*}{$\begin{array}{l}\text { No analgesic use } \\
\text { NSAIDs only } \\
\text { Paracetamol only } \\
\text { Both NSAIDs and } \\
\text { paracetamol }\end{array}$} & 38 & 37 & 1.0 & $1 \cdot 0$ & 36 & 1.0 \\
\hline & 30 & 50 & $0.55(0.3$ to 1.1$)$ & $0.59(0.3$ to 1.2$)$ & 45 & $0.71(0.4$ to 1.4$)$ \\
\hline & 69 & 51 & $1.26(0.7$ to $2 \cdot 3)$ & $1.50(0.7$ to 3.0$)$ & 77 & $1.03(0.6$ to 1.9$)$ \\
\hline & & & & & & \\
\hline & 10 & 15 & $0.61(0.2$ to 1.6$)$ & $0.73(0.3$ to 2.1$)$ & 18 & $0.57(0.2$ to 1.4$)$ \\
\hline
\end{tabular}




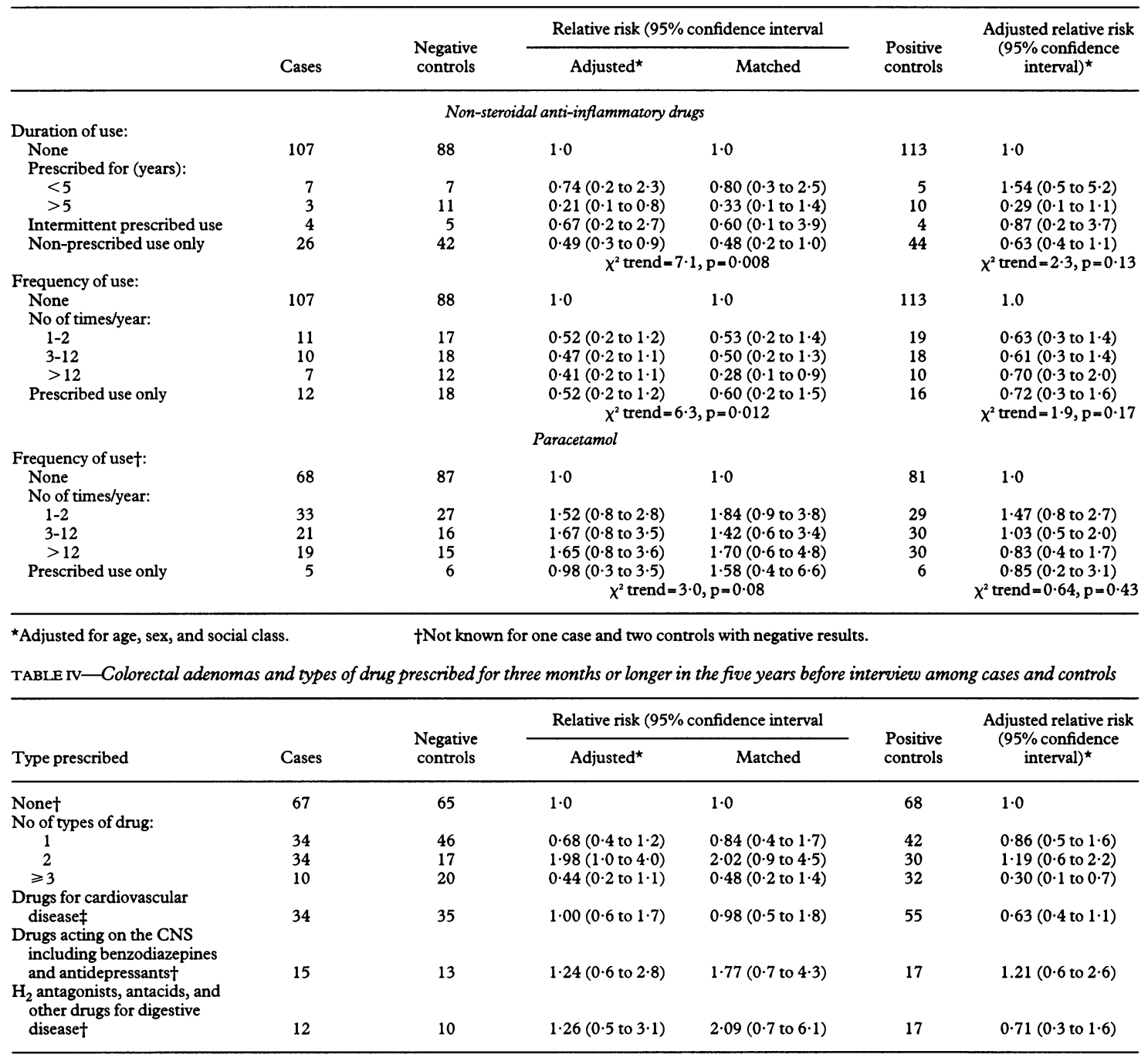

CNS = central nervous system.

*Adjusted for age, sex, and social class.

†Referent group; includes two cases, five negative controls, and four positive controls for whom number of types was not known.

use $\left(\chi^{2}\right.$ for trend $=7 \cdot 1, p=0.008$ and $\chi^{2}$ for trend $=2 \cdot 3$, $\mathrm{p}=0 \cdot 13$ on unmatched estimated relative risks for negative and positive controls respectively).

About two thirds of non-steroidal anti-inflammatory drug users in all three groups reported taking nonprescribed non-steroidal anti-inflammatory drugs, mainly aspirin. Two cases, five negative controls, and four positive controls reported taking non-prescribed ibuprofen. In comparison with the negative controls but not the positive controls the estimated relative risks declined with increasing frequency of use of nonprescribed non-steroidal anti-inflammatory drugs $\left(\chi^{2}\right.$ for trend $=6.3, p=0.012$ and $\chi^{2}$ for trend $=1.9, p=0.17$ on unmatched estimated relative risks for negative and positive controls respectively). In contrast, the presence of an adenoma and the frequency of paracetamol use was not significantly related in comparisons with either control group $\left(\chi^{2}\right.$ for trend $=3 \cdot 0, p=$ 0.08 and $\chi^{2}$ for trend $=0.64, p=0.43$ on unmatched estimated relative risks for negative and positive controls respectively).

\section{OTHER TYPES OF PRESCRIBED DRUG USE}

No other types of prescribed drug use showed a significant positive or negative association with having an adenoma (table IV). Overall, 78 (54\%) cases, 83 $(56 \%)$ negative controls, and $104(60 \%)$ positive controls reported having taken at least one prescribed drug for three months or longer over the previous five years.

A history of having had treatment for ulcers or indigestion that lasted for longer than two weeks, conditions which are relative contraindications to taking non-steroidal anti-inflammatory drugs, was reported by $53(36 \%)$ cases compared with $47(31 \%)$ negative controls and $52(30 \%)$ positive controls (estimated relative risk $1.3(0.8$ to 2.2$)$ and $1.4(0.9$ to $2 \cdot 3)$ respectively). However, only 11 cases, nine negative controls, and 15 positive controls reported taking $\mathrm{H}_{2}$ antagonists or antacids for three months or longer.

\section{CONSIDERATION OF CONFOUNDING}

Eight variables were identified as potential confounders in comparisons of cases with negative controls and seven variables in comparisons with the positive controls; only two were common to both sets of comparisons-namely, the total number of prescribed drugs and history of a parent having a stroke. None of the potential confounders was found to influence the results with the negative controls. Only the number of drugs had any effect on the results with positive controls, the estimated relative risk associated with more than five years of taking non-steroidal antiinflammatory drugs becoming $0.49(0.1$ to $2 \cdot 0)$ after adjustment.

\section{Discussion}

Our data support the hypothesis that taking aspirin or other non-steroidal anti-inflammatory drugs protects against the development of colorectal cancer and 
suggest that it does so by reducing the prevalence of colorectal adenomas. The association seems to be specific for non-steroidal anti-inflammatory drugs and is stronger for prolonged use. The consistency of the association within our study makes it unlikely that this is a chance result.

\section{BIAS AND CONFOUNDING}

Could bias or confounding account for these findings? The possibility that bias was introduced by the method of reporting or interviewing seems remote. At the time of interviewing we were unaware of the studies showing that non-steroidal anti-inflammatory drugs could influence experimental carcinogenesis and the drug data were collected in the middle of a lengthy interview mainly about diet. Indeed, our rationale for inquiring about analgesic use was the possibility that non-steroidal anti-inflammatory drug use might be associated with particular dietary factors as well as being a cause of positive results in a faecal occult blood test. Evidence is conflicting on how often aspirin and non-steroidal anti-inflammatory drugs are the cause of positive results in faecal occult blood test, although they can undoubtedly produce small increases in faecal blood loss. ${ }^{2426}$ Nevertheless, as our cases were identified from their positive results on testing they might have been expected to report greater rather than less use of aspirin and non-steroidal anti-inflammatory drugs compared with the controls with negative results on testing. Thus any bias introduced by selecting cases of adenoma with positive results in faecal occult blood tests should have produced differences opposite to those that we found in comparison with the negative controls.

How any selection bias might affect comparisons between the cases and the controls with positive results in a faecal occult blood test is more difficult to assess. Our reasons for recruiting these controls were twofold. Firstly, they would have been investigated and treated in a similar way to the cases. Secondly, investigation would have shown them to be free of colorectal adenomas, whereas at least $10 \%$ of the controls with negative results would be likely to have undetected colorectal adenomas. In retrospect most were found to have no obvious reason for their positive results. ${ }^{27}$ However, there was some evidence of greater morbidity in this group from its greater reported use of commonly prescribed drugs such as diuretics and $\beta$ blockers and a greater number of drugs being prescribed (table IV). How this might affect use of prescribed and nonprescribed non-steroidal anti-inflammatory drugs is debatable. The positive control group did contain some subjects with inflammatory bowel disease (12) and symptomatic diverticular disease (12), but analyses with these subjects excluded produced similar results. Depending on the proportion of colorectal adenomas that are truly bleeding rather than having been detected by serendipity and the proportion of positive results in faecal occult blood tests that are caused by use of non-steroidal anti-inflammatory drugs, the use of non-steroidal anti-inflammatory drugs might be expected to be higher in the positive controls than in the adenoma cases. ${ }^{28} 29$ Indeed, an earlier study in Nottingham found that $6 \%(7 / 113)$ of participants with colorectal adenomas compared with $13 \%$ (40/316) of those with positive results in a faecal occult blood test but no adenomas reported taking such drugs at the time of stool testing. ${ }^{30}$ In view of these considerations we think greater weight should be given to comparisons between cases and controls with negative results in faecal occult blood tests.

To assess potential confounding we examined the relation between use of non-steroidal antiinflammatory drugs and diet, physical activity, medical history, and other drug use and identified

\section{Clinical implications}

- Colorectal or bowel cancer is second only to lung cancer as a cause of cancer deaths

- Most bowel cancers develop from colorectal adenomas, a particular type of bowel polyp

- In this study people with colorectal adenomas reported less use of aspirin and less use of other non-steroidal anti-inflammatory drugs but not paracetamol compared with control subjects without such adenomas

- These results suggest that aspirin and other non-steroidal anti-inflammatory drugs reduce the development of adenomas and add to growing evidence that these drugs, even in modest doses, protect against colorectal cancer

no major confounding effects. Although taking aspirin occasionally was inversely associated with the development of adenomas, such infrequent use is unlikely to be protective and we ascribe this finding to the imprecision of our assessment of intake and the play of small numbers as shown by the wide confidence limits.

\section{MECHANISM OF PROTECTIVE EFFECT}

The mechanisms whereby non-steroidal antiinflammatory drugs might prevent the development of colorectal adenomas or cancer are not clear. It is notable that in familial adenomatous polyposis sulindac causes most polyps to shrink and disappear within six months. ${ }^{12-14}$ In experimental animals both indomethacin and piroxicam prevent the development of colonic cancer even when administered several weeks after the carcinogen has been given. ${ }^{78} \mathrm{~A}$ direct effect on reducing cellular proliferation seems unlikely because, if anything, non-steroidal anti-inflammatory drugs seem to increase the proliferative activity of colonic mucosa. ${ }^{31}$ As a major effect of non-steroidal anti-inflammatory drugs is inhibition of cyclooxygenase activity and thereby prostaglandin synthesis an effect via this pathway seems most likely but the mechanisms through which prostaglandins enhance experimental carcinogenesis are not established.

\section{CONCLUSIONS}

In conclusion, our data support the hypothesis that non-steroidal anti-inflammatory drug use protects against the development of colorectal neoplasia. The strength of the association is similar to that found in the three other epidemiological studies in which nonsteroidal anti-inflammatory drug use has been associated with a halving of the risk of colorectal cancer. Studies are now needed to confirm these findings, to determine how non-steroidal anti-inflammatory drugs might act, and particularly to see if non-steroidal antiinflammatory drugs can prevent the recurrence of adenoma or even cause sporadic adenomas to regress.

We acknowledge the support of the Cancer Research Campaign and the contribution of our interviewers, Gwyn Campion and Jenny Sterland.

1 Pollard M, Luckert PH. Indomethacin treatment of rats with dimethylhydrazine-induced intestinal tumors. Cancer Treat Rep 1980;64:1323-7.

2 Pollard M, Luckert PH, Schmidt MA. The suppressive effect of piroxicam on autochthonous intestinal tumors in the rat. Cancer Lett 1983;21:57-61.

3 Pollard M, Luckert PH. Treatment of chemically-induced intestinal cancers with indomethacin Proc Sac Exp Biol Med 1981:167:161-4.

4 Pollard M, Luckert PH. Prolonged antitumor effect on indomethacin on autochthonous intestinal tumors in rats. $₹$ Natl Cancer Inst 1983;70:1103-5.

5 Narisawa T. Sato M, Tani M, Kudo T, Takahashi T, Goto A. Inhibition of development of methylnitrosourea-induced rat colon tumors by indodevelopment of methylnitrosourea-induced rat

6 Narisawa $T$, Satoh $M$, Sano $M$, Takahashi $T$. Inhibition of initiation and promotion by $\mathrm{N}$-methylnitrosourea-induced colon carcinogenesis in rats by non-steroid anti-inflammatory agent indomethacin. Carcinogenesis 1983;4 1225-7. 
7 Reddy BS, Maruyama H, Kelloff G. Dose-related inhibition of colon carcinogenesis by dietary piroxicam, a nonsteroidal antinflammatory drug, during different stages of rat colon tumor development. Cancer Res 1987;47:5340-6.

8 Reddy BS, Nayini J, Tokumo K, Rigotty J, Zang E, Kelloff G. Chemoprevention of colon carcinogenesis by concurrent administration of piroxicam, a nonsteroidal antinflammatory drug with $D, L-\alpha$-difluoromethylornithine, an ornithine decarboxylase inhibitor, in diet. Cancer Res 1990;50:2562-8.

9 Metzger U, Meier J, Uhlschmid G, Weihe H. Influence of various prostaglandin synthesis inhibitors on DMH-induced rat colon cancer. Dis Colon Rectum 1984;27:366-9.

10 Waddell WR, Loughry RW. Sulindac for polyposis of the colon. I Surg Oncol 1983;24:83-7.

11 Labayle D, Fischer D, Vielh P, Drouhin F, Pariente A, Bories C, et al. Sulindac causes regression of rectal polyps in familial adenomatous polyposis. Gastroenterology 1991;101:635-9.

12 Nugent KP, Farmer KCR, Spigelman AD, Williams CB, Phillips RKS Randomised controlled clinical trial of Sulindac on intestinal polyposis in FAP. Gut 1992;33:S33.

13 Giardiello FM, Hamilton SR, Krush AJ, Piantadosi S, Hylind LM, Celano P, et al. Treatment of colonic and rectal adenomas with sulindae in familial adenomatous polyposis. N Engl f Med 1993;328:1313-6.

14 Kune GA, Kune S, Watson LF. Colorectal cancer risk, chronic illnesses, operations, and medications: case control results from the Melbourne Colorectal Cancer Study. Cancer 1988;48:399-404.

15 Rosenberg L, Palmer JR, Zauber AG, Warshauer ME, Stolley PD, Shapiro S. A hypothesis: nonsteroidal anti-inflammatory drugs reduce the incidence of large bowel-cancer. $\mathcal{F}$ Natl Cancer Inst 1991;83:355-8.

16 Thun MJ, Namboodiri MM, Heath CW. Aspirin use and reduced risk of colon cancer. N Engl f Med 1991;325:1593-6.

17 Paganini-Hill A, Hsu G, Ross RK, Henderson BE. Aspirin use and incidence of large-bowel cancer in a California retirement community. $\mathcal{f}$ Natl Cancer Inst 1991;83:1182-3.

18 Little J, Logan RFA, Hawtin PG, Hardcastle JD, Turner ID. Colorectal adenomas and diet: a case-control study of subjects participating in the Nottingham faecal occult blood screening programme. Br 9 Cancer 1993;67:177-84.
19 Hardcastle JD, Thomas WM, Chamberlain J, Pye G, Sheffield J, James PD, et al. Randomised, controlled trial of faecal occult blood screening for col. Randomised, cont Cock the Cancer, 1988 .

21 Baker RJ, Clarke MRB, Nelder JA. GLIM: the generalised linear interactive modelling system. Oxford: Numerical Algorithms Group, 1985. (GLIM 3.77 Manual and Macro Library Release 1.1.)

22 Statistics and Epidemiology Research Corporation. EGRET (Epidemiological Graphics, Estimation, and Testing Package). Seattle: SERC, 1991.

23 Armitage N, Hardcastle JD, Amar SS, Balfour TW, Haynes J, James PD. A comparison of an immunological faecal occult blood test Fecatwin sensitive/ FECA EIA with Haemoccult in population screening for colorectal cancer. Brf Cancer 1985;51:799-804.

24 Rees WD, Turnberg LA. Reappraisal of the effects of aspirin on the stomach. Lancet 1980;ii:410-3.

25 Doran J, Hardcastle JD. Bleeding patterns in colorectal cancer: the effect of aspirin and the implications for faecal occult blood testing. $\mathrm{Br} f$ Surg 1982;69:711-3.

26 Norfleet RG. 1,300 mg of aspirin daily does not cause positive fecal Hemoccult tests. $\mathcal{F C l i n}$ Gastroenterol 1983;5:123-5.

27 Thomas WM, Hardcastle JD. Role of upper gastrointestinal investigations in a screening study for colorectal neoplasia. Gut 1990;31:1294-7.

28 Ransohoff DF, Lang CA. Small adenomas detected during fecal occult blood test screening for colorectal cancer: the impact of serendipity. $\mathscr{f} A M A$ 1990;264:76-8.

29 Simon JB. Colonic polyps, occult blood, and chance. FAMA 1990;264:84-

30 Pye G, Ballantyne KC, Armitage NC, Hardcastle JD. Influence of nonsteroidal anti-inflammatory drugs on the outcome of faecal occult blood tests in screening for colorectal cancer. $B M 7$ 1987;294:1510-1.

31 DeRubertis FR, Craven PA, Saito R. 16,16-Dimethyl prostaglandin E suppresses the increases in the proliferative activity of rat colonic epithelium induced by indomethacin and aspirin. Gastroenterology 1985;89:1054-63.

(Accepted 25 May 1993)

\section{INSERM U 360,}

\section{Recherches}

Epidémiologiques en

Neurologie et

Psychopathologie,

Villejuif, France

Christophe Tzourio, senior

registrar

Jean-Baptiste Hubert, research fellow

Annick Alpérovitch, director of research

Service de Neurologie, Hôpital Saint-Antoine,

Paris, France

Serge Iglesias, registrar

Alain Tehindrazanarivelo,

senior registrar

Valérie Biousse, registrar

Marie-Germaine Bousser,

professor of neurology

Service de Neurologie, Hôpital Lariboisière, Paris, France

Jean-Marc Visy, senior

registrar

France Woimant, consultant

Correspondence to:

Dr C Tzourio, INSERM

U 360, Recherches

Epidémiologiques en

Neurologie et

Psychopathologie, 16 av

Paul Vaillant Couturier,

Cedex 94807 Villejuif,

France.

$B M F$ 1993;307:289-92

\title{
Migraine and risk of ischaemic stroke: a case-control study
}

\author{
Christophe Tzourio, Serge Iglesias, Jean-Baptiste Hubert, Jean-Marc Visy, Annick Alpérovitch,
} Alain Tehindrazanarivelo, Valérie Biousse, France Woimant, Marie-Germaine Bousser

\section{Abstract}

Objectives-To determine whether migraine is a risk factor for ischaemic stroke.

Design-A case-control study.

Setting-Two hospitals in Paris.

Subjects-212 patients with stroke (137 men and 75 women) and 212 controls matched for sex, age (to within five years), and history of hypertension.

Main outcome measures-Ischaemic stroke, confirmed by brain computed tomography or magnetic resonance imaging, and history of headache, recorded with structured questionnaire during interview.

Results-Prevalence of migraine did not differ between patients with stroke and controls: 18/137 $v$ $17 / 137$ for men (odds ratio 1.1 (95\% confidence interval 0.5 to $2 \cdot 2$ ), $p=0.86) ; 23 / 75 v 17 / 75$ for women (odds ratio $1.6(0.7$ to 3.5$), \mathrm{p}=0.24)$; and $41 / 212 v 34 /$ 212 for both sexes (odds ratio $1.3(0.8$ to $2 \cdot 3), p=$ $0.33)$. When subjects were split into two age groups, however, prevalence of migraine was significantly higher among younger women (aged <45) with stroke compared with their controls $(13 / 20 v 6 / 20$, odds ratio $4.3(1.2$ to $16 \cdot 3), p=0.03)$. Furthermore, the risk of ischaemic stroke was higher among younger women who smoked $(7 / 20 v 1 / 20$, odds ratio $10 \cdot 2(1 \cdot 1$ to $93 \cdot 3))$.

Conclusions-Prevalence of migraine was not different between patients with stroke and matched controls except among women aged $<45$, when migraine and stroke were significantly associated.

\section{Introduction}

Besides being a rare but well documented cause of cerebral infarction - migrainous infarct-migraine has episodically been suggested as a risk factor for stroke. Indeed, the vasomotor changes ${ }^{1}$ and modifications of platelet aggregability ${ }^{2}$ that occur during migraine could cause an ischaemic stroke. Few epidemiological studies have addressed the question of the link between migraine and stroke, and their results are not consistent. As examples of the wide range of values observed, the risk of stroke associated with migraine was $1 \cdot 2$ for women aged under 45 not using oral contraceptives ${ }^{4}$ and was 31.3 for a subgroup of patients without vascular risk factors. ${ }^{5}$

The present study was performed to address this issue and was the first to be based on the diagnostic criteria for migraine recently proposed by the International Headache Society. ${ }^{6}$

\section{Patients and methods}

All patients aged $18-80$ successively admitted to two Paris hospitals with a diagnosis of ischaemic stroke over 24 months were included in the study provided they were willing and able to answer the questionnaire. Ischaemic stroke was defined clinically, according to the World Health Organisation's criteria, ${ }^{7}$ and was confirmed in all patients by computed tomography or magnetic resonance imaging of the brain. Patients with transient ischaemic attacks or cerebral venous thrombosis were excluded. Controls were selected from patients admitted to the hospitals with benign rheumatological conditions or for routine surgical procedures excluding vascular surgery. Patients with a history of stroke were excluded. Controls were matched with patients with stroke for age (to within five years), sex, and history of hypertension.

Each subject's history of headache was recorded during an interview in hospital by a neurologist with a highly structured questionnaire. This covered all of the International Headache Society's diagnostic criteria for migraine with or without aura and different subtypes. ${ }^{6}$ The subjects were not aware of the final aim of the study: they were just asked if they wanted to participate in a neuroepidemiological study, and none 